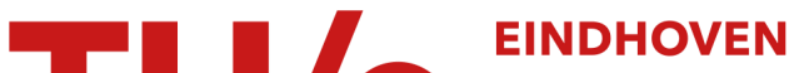 \\ UNIVERSITY OF \\ TECHNOLOGY
}

\section{The behavior of adaptive bone-remodelling simulation models}

Citation for published version (APA):

Weinans, H., Huiskes, H. W. J., \& Grootenboer, H. J. (1992). The behavior of adaptive bone-remodelling simulation models. Journal of Biomechanics, 25(12), 1425-1441. https://doi.org/10.1016/0021-9290(92)90056-7

DOI:

10.1016/0021-9290(92)90056-7

Document status and date:

Published: 01/01/1992

\section{Document Version:}

Publisher's PDF, also known as Version of Record (includes final page, issue and volume numbers)

\section{Please check the document version of this publication:}

- A submitted manuscript is the version of the article upon submission and before peer-review. There can be important differences between the submitted version and the official published version of record. People interested in the research are advised to contact the author for the final version of the publication, or visit the $\mathrm{DOI}$ to the publisher's website.

- The final author version and the galley proof are versions of the publication after peer review.

- The final published version features the final layout of the paper including the volume, issue and page numbers.

Link to publication

\section{General rights}

Copyright and moral rights for the publications made accessible in the public portal are retained by the authors and/or other copyright owners and it is a condition of accessing publications that users recognise and abide by the legal requirements associated with these rights.

- Users may download and print one copy of any publication from the public portal for the purpose of private study or research.

- You may not further distribute the material or use it for any profit-making activity or commercial gain

- You may freely distribute the URL identifying the publication in the public portal.

If the publication is distributed under the terms of Article 25fa of the Dutch Copyright Act, indicated by the "Taverne" license above, please follow below link for the End User Agreement:

www.tue.nl/taverne

Take down policy

If you believe that this document breaches copyright please contact us at:

openaccess@tue.nl

providing details and we will investigate your claim. 


\title{
THE BEHAVIOR OF ADAPTIVE BONE-REMODELING SIMULATION MODELS
}

\author{
H. WEINANS, ${ }^{*}$ R. HUISKES* and H. J. GROOTENBOER $\dagger$ \\ *Biomechanics Section, Institute of Orthopaedics, University of Nijmegen, P.O. Box 9101, $6500 \mathrm{HB}$ \\ Nijmegen, The Netherlands and $\uparrow$ Department of Fundamental Mechanical Engineering, Faculty of \\ Mechanical Engineering, University of Twente, The Netherlands
}

\begin{abstract}
The process of adaptive bone remodeling can be described mathematically and simulated in a computer model, integrated with the finite element method. In the model discussed here, cortical and trabecular bone are described as continuous materials with variable density. The remodeling rule applied to simulate the remodeling process in each element individually is, in fact, an objective function for an optimization process, relative to the external load. Its purpose is to obtain a constant, preset value for the strain energy per unit bone mass, by adapting the density. If an element in the structure cannot achieve that, it either turns to its maximal density (cortical bone) or resorbs completely.

It is found that the solution obtained in generally a discontinuous patchwork. For a two-dimensional proximal femur model this patchwork shows a good resemblance with the density distribution of a real proximal femur.

It is shown that the discontinuous end configuration is dictated by the nature of the differential equations describing the remodeling process. This process can be considered as a nonlinear dynamical system with many degrees of freedom, which behaves divergent relative to the objective, leading to many possible solutions. The precise solution is dependent on the parameters in the remodeling rule, the load and the initial conditions. The feedback mechanism in the process is self-enhancing; denser bone attracts more strain energy, whereby the bone becomes even more dense. It is suggested that this positive feedback of the attractor state (the strain energy field) creates order in the end configuration. In addition, the process ensures that the discontinuous end configuration is a structure with a relatively low mass, perhaps a minimal-mass structure, although this is no explicit objective in the optimization process.

It is hypothesized that trabecular bone is a chaotically ordered structure which can be considered as a fractal with characteristics of optimal mechanical resistance and minimal mass, of which the actual morphology depends on the local (internal) loading characteristics, the sensor-cell density and the degree of mineralization.
\end{abstract}

\section{INTRODUCTION}

It is assumed that the morphology of bone relates to its internal mechanical loads. The process which regulates this relationship is called bone modeling or remodeling. It is believed that bone has the ability to adapt itself to the loading conditions to which it is exposed. This implies that bone must have internal sensors, to measure the internal load, and transducers releasing signals and activating cells, to resorb or form bone. The change of net bone mass can be accomplished by a modification of either density or geometry.

Several attempts to quantify the bone-remodeling process have been reported in the literature. Following the qualitative observations of Wolff (1892), several investigators tried to describe the process mathematically, in order to accurately predict bone formation or resorption (Frost, 1964a,b; Pauwels, 1965; Kummer, 1972; Cowin and Hegedus, 1976; Frost, 1987). For qualitative predictions it is necessary that the internal mechanical load in the bone structure can

Received in final form 15 March 1992.

Partly presented at the Orthopaedic Research Society, 35th Annual Meeting, Las Vegas, U.S.A. (1989) and at the First World Congress of Biomechanics, San Diego, U.S.A. (1990). be determined accurately in terms of stresses and strains, for which the finite element method (FEM) is an effective tool (Huiskes and Chao, 1983). By combining mathematical bone-remodeling descriptions with finite element models, quantitative predictions about bone formation and resorption in realistic bone structures can be made (Hart et al., 1984; Fyhrie and Carter, 1986; Huiskes et al., 1987, 1989a,b, 1991; Carter et al., 1989; Weinans et al., 1989, 1990; Beaupré et al. 1990b). These models are all based on the principle that bone remodeling is induced by a local mechanical signal which activates the regulating cells (osteoblasts and osteoclasts). Hence, it is assumed that the bone has sensors, which can detect a mechanical stimulus and, depending on the magnitude of this stimulus, cause local bone adaptations. This process can be described with a generic mathematical expression, using the apparent density as the characterization of the internal morphology. The rate of change of the apparent density of the bone at a particular location $\mathrm{d} \rho / \mathrm{d} t$, with $\rho=\rho(x, y, z)$, can be described as an objective function $F$, which depends on a particular stimulus at location $(x, y, z)$. It is assumed that this stimulus is directly related to the local mechanical load in the bone and can be determined from the local stress tensor $\sigma=\sigma(x, y, z)$, the local strain tensor 
$\varepsilon=\varepsilon(x, y, z)$, and the apparent density $\rho=\rho(x, y, z)$ :

$$
\frac{\mathrm{d} \rho}{\mathrm{d} t}=F(\sigma, \varepsilon, \rho), \quad 0<\rho \leqslant \rho_{\mathrm{cb}},
$$

where $\rho_{c \mathrm{~b}}$ is the maximal density of cortical bone. When the objective function $F$ reaches zero, the system is in equilibrium and the net bone-density rate of change is zero.

Such a generic relationship can be specified to

$$
\frac{\mathrm{d} \rho}{\mathrm{d} t}=B(S-k), \quad 0<\rho \leqslant \rho_{\mathrm{cb}},
$$

where $B$ is a constant, $S=S(x, y, z)$ is the mechanical stimulus and $k=k(x, y, z)$ is a constant. When combined with a FEM, $S$ is usually expressed per element. In that case it is, in fact, assumed that there is precisely one sensor point per element. Equation (2) signifies that the stimulus strives to become equal to the reference value $k$, which can either be site-specific $[k=k(x, y, z)]$ or non-site-specific ( $k=$ constant).

Cowin and Hegedus (1976) proposed a relationship in which the adaptation of the elastic modulus of the bone was coupled directly to deviations of the strain tensor. Hence, the individual components of the actual strain tensor were considered as stimuli, and a difference between the actual strain values and reference values at the same location would cause adaptations in moduli. Huiskes et al. (1987) used a similar approach but, instead of the strain tensor, the strain energy density (SED) was considered as the stimulus. A difference between the actual SED and a reference SED at the same location would then be the driving force for adaptation of the apparent density. Hence, in equation (2), $S=S(x, y, z)$ would be the actual SED and $k=k(x, y, z)$, the reference SED. Both these theories are 'site-specific' because the reference state of the stimulus depends on location. This implicates that the normal stimulus distribution $k=k(x, y, z)$ must be known or be determined from a normal equilibrium density distribution, in order to predict the bone adaptation process to an abnormal situation, e.g. after a change in loading, geometry or placement of an implant.

The original hypotheses of Wolff (1892), however, have much stronger implications than just the notion that bone would remodel to deviations in its normal stress environment. Based on the ideas of Roux (1881), Wolff assumed bone to be a material with self-optimizing capabilities and able to control its mass and structure in direct relationship to its mechanical demands. Fyhrie and Carter (1986) proposed a theory, suitable, in principle, to describe such a self-optimizing process mathematically. They postulated that bone would adopt its apparent density (or porosity) locally for any loading environment, in order to normalize a predestined 'effective stress' value. This 'bone-maintenance theory' (Carter et al., 1989) was later specified to a particular form in which the strain energy density (SED) in the trabeculae was sub- stituted in the 'effective stress' criterion (Fyhrie and Carter, 1990). This SED value in the trabeculae was approximated by $U / \rho$, where $U$ is the apparent SED in the bone when it is assumed continuous and $\rho$ is the apparent density. Hence, $U / \rho$ represents the strain energy per unit of bone mass. In their theory of self-optimization or bone maintenance, they also took account of variable loading, defining the stimulus from a loading history in terms of a SED magnitude calculated from each of the individual loading configurations $F_{i}$ and the total number $n$ of these loading configurations considered. Hence,

$$
\frac{1}{n} \sum_{i=1}^{n}\left(\frac{U_{i}}{\rho}\right)=\text { constant }
$$

is the goal of the remodeling process, whereby $U_{i}=U_{i}(x, y, z)$ is apparent SED for loading case $i$. Here again, it must be mentioned that this goal is only valid for $0<\rho \leqslant \rho_{\mathrm{cb}}$.

Such a remodeling objective can also be incorporated in a time-dependent remodeling rule such as equations (1) and (2) (Weinans et al., 1988; Huiskes et al., 1989a,b; Beaupré et al., 1990a):

$$
\frac{\mathrm{d} \rho}{\mathrm{d} t}=B\left(\frac{U_{\mathrm{a}}}{\rho}-k\right), \quad 0<\rho \leqslant \rho_{\mathrm{cb}}
$$

where $\rho=\rho(x, y, z)$ is the apparent density, $B$ and $k$ are constants, and

$$
U_{\mathrm{a}}=\frac{1}{n} \sum_{i=1}^{n} U_{i}
$$

Relative to the more conservative site-specific approach, this formulation has much stronger consequences because it is suitable to predict bone density for any geometric or loading configuration, in accordance with Wolf's law. The role of the 'evolution' is represented by the value of only one constant, $k$, which is non-site-specific. This process is considered to be converged when $\mathrm{d} \rho / \mathrm{d} t$ is zero according to equation (3) or when the density has reached its minimal or maximal value (completely resorbed or $\rho=\rho_{\mathrm{cb}}$ ). Bonedensity distributions of the proximal femur predicted with this remodeling rule in combination with FEM models turned out to be more or less realistic (Carter et al., 1989; Huiskes et al, 1987, 1989a,b; Weinans et al., 1989, 1990; Beaupré et al., 1990b).

The purpose of the present study was to obtain a better understanding of the behavior of the strainadaptive bone-remodeling simulation rule represented by equation (3) in combination with FEM models. In particular, the stability and convergence behavior of the remodeling rule were investigated in relation to the characteristics of the FE mesh. It is important to realize that the remodeling rule of equation (3) is usually applied per element. Hence, mesh refinement automatically implies an increase in sensor (cell) density. 


\section{METHOD}

In this paper we assume the non-site specific formulation of adaptive bone remodeling as specified in equation (3). The stimulus $S\left(=U_{\mathrm{a}} / \rho\right)$ is, as a rule, measured per element, indicating one sensor per element, and the apparent density is also adapted per element.

Three different structures were considered to study the simulation process: (i) a proximal femur; (ii) a square plate; and (iii) a two-unit model with two sensors only.

For the finite element procedure the MARC finite element code is used (Marc Analysis Research Corporation, Palo Alto, CA). The remodeling formulation and the FEM code are integrated as shown in Fig. 1.

\section{The proximal femur model}

A two-dimensional finite element model of a proximal femur was constructed as shown in Fig. 2. A sideplate connects the periosteal sides of the medial and lateral cortices in order to account for the threedimensional structural integrity of the bone (Huiskes, 1980; Verdonschot and Huiskes, 1990). The remodeling rule was applied to the front-plate only. The Young's modulus of the side-plate remains unchanged at 17,000 MPa. The Poisson's ratio for both the front and the side-plate was taken as 0.3 . As proposed by Carter et al. (1989), three loading cases were used (Fig. 2); hence, $U_{\mathrm{a}}$ in equation (3) is determined from these three loading cases. The reference value $k$ is taken as $0.004\left[\mathrm{MPa}\left(\mathrm{g} \mathrm{cm}^{-3}\right)^{-1}=\mathrm{Jg}^{-1}\right]$, since experience with this model showed that this value leads to a realistic end configuration (Weinans et al., 1989; Huiskes et al., 1989b).

The relation between density and Young's modulus was taken from Carter and Hayes (1977) as $E=3790 \rho^{3}$. The minimal density was $0.01 \mathrm{~g} \mathrm{~cm}^{-3}$, representing complete resorption of an element. The maximal density $\left(\rho_{\mathrm{cb}}\right)$ was taken as $1.74 \mathrm{~g} \mathrm{~cm}^{-3}$, which corresponds to a Young's modulus of almost 20,000 MPa.

Each element (sensor or sample point) has, in principle, three possibilities to converge and, hence, reach the remodeling equilibrium: (1) the bone is completely resorbed $\left(\rho=0.01 \mathrm{~g} \mathrm{~cm}^{-3}\right)$; (2) the bone becomes cortical $\left(\rho=\rho_{\mathrm{cb}}=1.74 \mathrm{~g} \mathrm{~cm}^{-3}\right)$; or (3) the bone remains cancellous with an apparent density satisfying equation (3) and, hence, $U_{\mathrm{a}} / \rho=k$ (with $k=0.004 \mathrm{~J} \mathrm{~g}^{-1}$ ). In all simulation analysis the process starts with a uniform density distribution of $0.8 \mathrm{~g} \mathrm{~cm}^{-3}$. Equation (3), including its boundary values for the maximal and minimal density, is solved with forward Euler integration using constant time steps; hence, $\Delta \rho=B \Delta t\left(U_{\mathrm{a}} / \rho-k\right)$, with $\Delta t=30.0$ in arbitrary time units and $B=1.0\left(\mathrm{~g} \mathrm{~cm}^{-3}\right)^{2}(\mathrm{MPa} \times$ time unit) ${ }^{-1}$. The time step was varied to guarantee that it was small enough not to affect the end result in a significant way (Weinans et al., 1989).

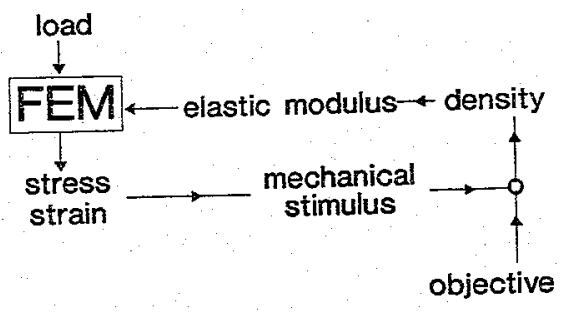

Fig. 1. The iterative feedback mechanism of a FEM-integrated computer simulation model of mechanically induced bone remodeling.

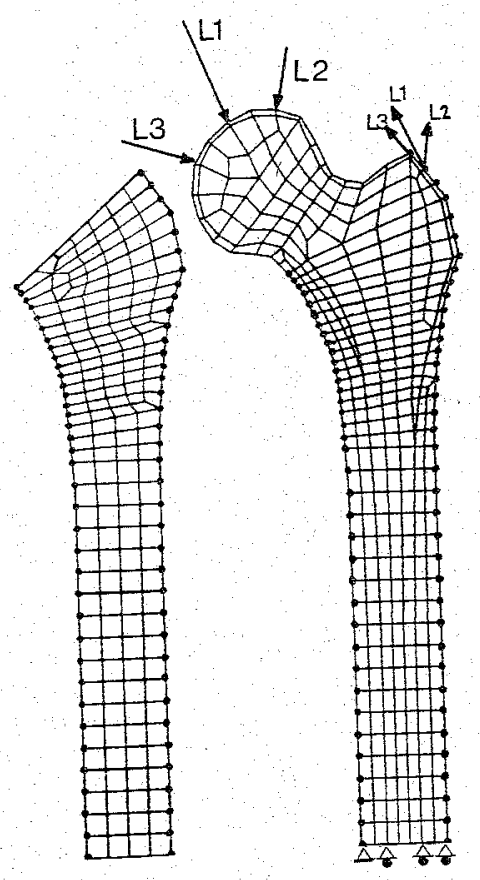

Fig. 2. Finite element model of proximal femur with sideplate. The side-plate (left) is connected to the front-plate (right) at the medial and lateral side (marked with thick nodes). The front-plate has a uniform thickness of $12 \mathrm{~mm}$ and the side-plate a nonuniform thickness, decreasing from $4 \mathrm{~mm}$ at the distal end to $0.4 \mathrm{~mm}$ at the proximal end. All elements are isoparametric with four nodes and a linear displacement field. L1, L2 and L 3 refer to loading cases $i=1$, 2 and 3.

\section{The plate model}

A simple two-dimensional FEM model of a square plate was constructed (Fig. 3). Mesh refinement was varied as indicated in the figure. Equation (3) was again used to control the remodeling process. The relation between Young's modulus $E$ and the apparent density was taken as

$$
E=c \rho^{\gamma}
$$

with $\gamma=2, c=100$ (MPa) $\left(\mathrm{g} \mathrm{cm}^{-3}\right)^{-2}$ and a Poisson's ratio of 0.3 . The constants in equation (3) were taken as $B=1.0\left(\mathrm{~g} \mathrm{~cm}^{-3}\right)^{2}(\mathrm{MPa} \times \text { time unit })^{-1}$ and 
$k=0.25\left(\mathrm{~J} \mathrm{~g}^{-1}\right)$. The time step in the Euler integration was taken as 1.0 arbitrary time units, and again it was checked whether this time step was small enough not to influence the solution process in a significant way. The parameters and load (Fig. 3) are chosen such that a representative example of a density distribution is obtained.

First, the effect of element type was investigated. For that purpose an eight-node element with a quadratic displacement field was tested against a four-node element with a bilinear displacement field. Next, the pure effects of element density (without the effects of sensor density) were investigated. New subdivisions of four or 16 elements within the elements of the $5 \times 5$ mesh were made (Fig. 3). These four or 16 elements were remodeled as a group (one sensor only) at the level of the $5 \times 5$ element mesh. Hence, in one sensor (with the location according to the $5 \times 5$ mesh) the SED is determined by taking the average from the group of four or 16 elements.

Finally, the combined effects of mesh refinement and the corresponding sensor density refinement were studied by increasing the number of elements in the model from $5 \times 5$ to $10 \times 10,20 \times 20$ and $40 \times 40$, each time with a sensor in each element.

\section{The two-unit model}

The two-unit model (Fig. 4) is developed to study the stability of the remodeling simulation process analytically, using closed-form solutions. Each unit (or element) is loaded by a compressive force, $P_{1}$ and $P_{2}$, respectively (Fig. 4), in such a way that the total force $P=P_{1}+P_{2}$ and the strains in the elements are equal, i.e. $\varepsilon_{1}=\varepsilon_{2}=\varepsilon$. The remodeling rule [equation (3)] is applied for each element separately; hence, each unit is assumed to have one sensor. Equation (4) is applied to relate density to elastic modules. The derivation of the analytical solution is given in the

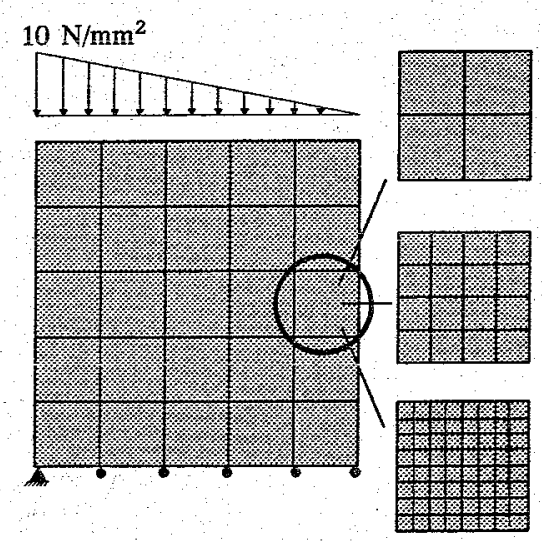

Fig. 3. Plate of $100 \times 100 \mathrm{~mm}$ with $5 \times 5$ elements (linear, with four nodes). To study the effects of element density, subdivisions of 4 or 16 elements within the $5 \times 5$ mesh were made. Mesh refinement and the corresponding sensor density refinement up to $40 \times 40$ elements was also carried out. appendix. Apart from a stimulus $S=U / \rho$, other possible kinds of stimuli are investigated as well.

\section{Convergence}

The convergence behavior of all three models was investigated. For this purpose, the objective function $\Theta$ is defined, for all three models, as

$$
\Theta=\frac{1}{m} \sum_{i=1}^{m}\left|\frac{U_{\mathrm{a}}^{i}}{\rho^{i}}-k\right|
$$

where $m$ is the number of elements and $U_{\mathrm{a}}^{i}$ is again the average SED for all loading cases considered in element $i$. The value of $\Theta$ is calculated after each iteration and indicates the extent to which the objective $\left(U_{\mathrm{a}} / \rho=k\right)$ is reached in the whole structure. It must be noted again that in those elements in which the bone resorbs completely $(\rho=0.01)$ or in which cortical bone is obtained $\left(\rho=\rho_{\mathrm{cb}}\right)$, the remodeling process stops; hence, the remodeling objective $U_{\mathrm{a}} / \rho=k$ will not be met.

In addition, the total mass $(M)$ of the structure is calculated after every iterative time step, from

$$
M=\sum_{i=1}^{m}\left(V^{i} \rho^{i}\right)
$$

where $V^{i}$ and $\rho^{i}$ are the volume and density, respectively, of element $i$, and $m$ is the number of elements in the structure.

\section{RESULTS}

\section{Femur model}

The results of the remodeling simulation of the femur model are presented in Fig. 5(a). The process was continued until virtually no more density changes occurred. Hence, $\mathrm{d} \rho / \mathrm{d} t$ was zero in virtually all elements. The end configuration predicts a reasonably accurate density distribution, with an intramedullary canal, Ward's triangle and the typical cancellous density patterns in the femoral head [compare with Fig. 5(b)].

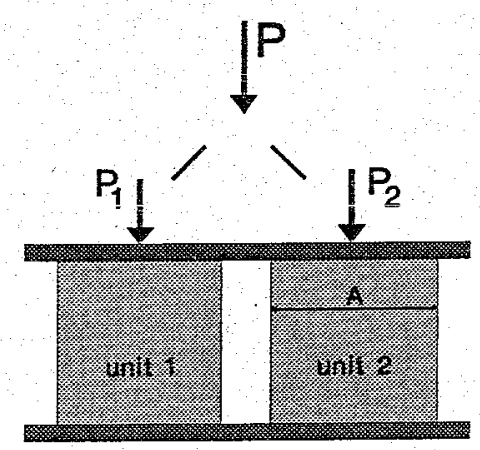

Fig 4. Example problem with two units. The total load is divided over the two units such that the deformation in each unit is the same $\left(P=P_{1}+P_{2}\right.$ and $\left.\varepsilon_{1}=\varepsilon_{2}=\varepsilon\right)$. $A$ is the crosssectional area in the units. 

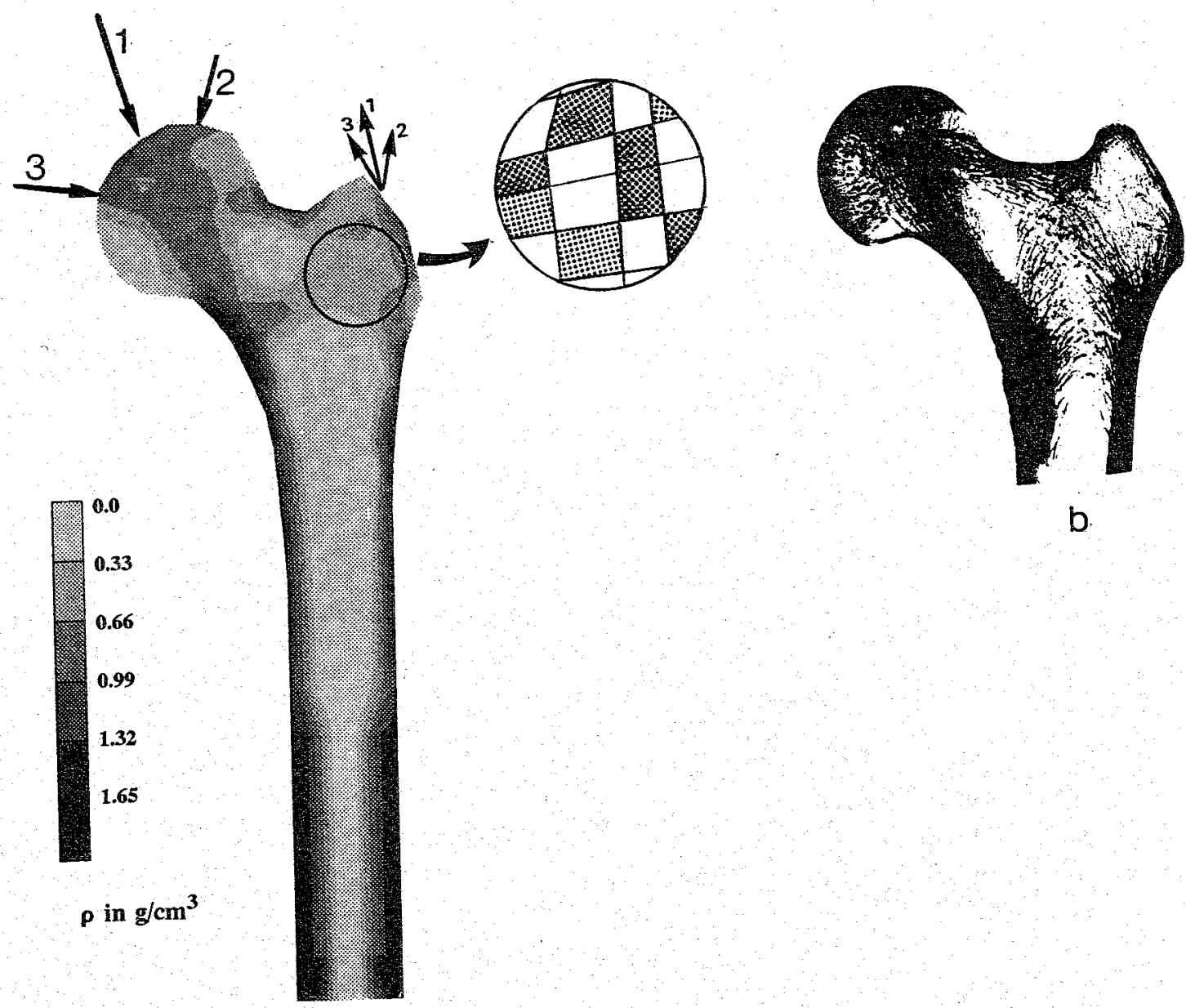

a

Fig. 5. (a) Density distribution from a remodeling simulation of a femur, starting from a uniform density distribution of $0.8 \mathrm{~g} \mathrm{~cm}^{-3}$ and a reference stimulus value of $k=0.004 \mathrm{Jg}^{-1}$. In the circle the density distribution is shown as it is found by the model without interpolation. (b) Morphology of a real femur.

A more detailed examination of the density patterns in the end configurations shows, however, that there are many empty elements in the seemingly continuous distribution, as shown in the enlargement of a region of Fig. 5(a) showing the real element density values without interpolation. It appears that most of the elements converge to the boundaries of the solution space and, hence, either to dense cortical bone $\left(\rho_{\mathrm{cb}}\right)$ or to the minimal density of $\rho=0.01$, representing complete resorption. The postprocessor of the FEM code, however, interpoiates these values in such a way that a continuous distribution results. In the postprocessing program, first the nodal-point density values per element are calculated by extrapolating from the integration-point values, which are then averaged over the connecting elements. Then the density values over the whole mesh are interpolated from the nodal-point values. Hence, a discontinuous solution is presented as continuous after postprocessing. This is a typical procedure for any FE code (compare Carter et al., 1989; Beaupré et al., 1990b). These dis- continuities will be further discussed below in relation to the other models.

It is found that the size of the time step is of minor influence to the predicted end configuration, as long as it is sufficiently small. This aspect will be discussed later in more detail.

The development of the objective function $\Theta$ over time is shown in Fig. 6, as integrated over all elements, and as integrated over the cancellous elements only, for which $0.01<\rho<\rho_{\mathrm{cb}}$. The former does not converge to zero, because of the elements which converge to the boundary density values, but the latter does. This indicates that indeed the process has fully converged.

\section{The two-unit model}

The remodeling behavior of the two-unit model is governed by a set of differential equations:

$$
\frac{\mathrm{d} \rho_{1}}{\mathrm{~d} t}=B\left(S_{1}-k\right) \text { and } \frac{\mathrm{d} \rho_{2}}{\mathrm{~d} t}=B\left(S_{2}-k\right)
$$


where $S_{1}=U_{1} / \rho_{1}$ and $S=U_{2} / \rho_{2}$. The SED, $U_{1}$ and $U_{2}$, depend only on the forces, $P_{1}$ and $P_{2}$, and on the apparent densities, $\rho_{1}$ and $\rho_{2}$, respectively: Since $P=P_{1}+P_{2}=$ constant, and $\varepsilon_{1}=\varepsilon_{2}=\varepsilon$, the forces $P_{1}$ and $P_{2}$ can also be expressed as functions of $\rho_{1}$ and $\rho_{2}$. Hence, the remodeling rates $\mathrm{d} \rho_{1} / \mathrm{d} t$ and $\mathrm{d} \rho_{2} / \mathrm{d} t$ in equation (7) can be expressed as nonlinear functions of $\rho_{1}$ and $\rho_{2}$ exclusively (see Appendix).

The behavior of the system is governed by a set of nonlinear differential equations, typical for nonlinear dynamical systems, with stable and unstable solutions. It appears (see Appendix) that the stability of the solution depends on the value of the exponent $\gamma$ from the relationship $E=c \rho^{\gamma}$ [equation (4)]. Only if $1-\gamma^{2}>0$ (hence, $|\gamma|<1.0$ ), we may obtain a stable uniform solution for which $\rho_{1}=\rho_{2}$ (a 'continuous' solution). When $\gamma>1.0$, which is the case for cancellous bone; a stable uniform (or 'continuous') solution does not exist. The outcome for $\gamma=1.0$ is uncertain.

Figure 7(a) shows the phase plot for the solution of the differential equations (7) when $\gamma>1.0$. Evidently,

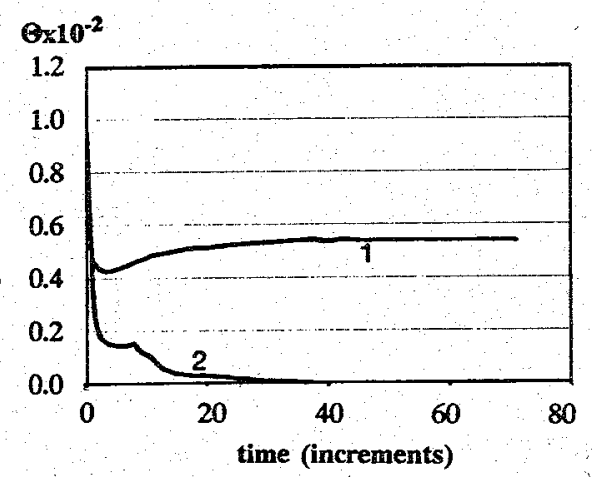

Fig. 6. The objective function $\Theta$ [equation (5)] during the remodeling process of the normal femur (Fig. 5). Line 1 indicates $\Theta$ as integrated over all elements and line 2 as integrated only over the cancellous bone elements with $0<\rho<\rho_{\mathrm{cb}}$ the solution $\rho_{1}=\rho_{2}=\rho_{e}$ represents an instable saddle point. It may be approached closely during an iterative process, depending upon the starting configuration, but continuing iteration will finally bring the process to either of the stable solutions $\rho_{1}=0$, $\rho_{2}=\rho_{\mathrm{m}}$, or $\rho_{1}=\rho_{\mathrm{m}}, \rho_{2}=0$. This is also illustrated in Fig. 7(b), where the course in time of $\rho_{1}$ and $\rho_{2}$ are shown. Starting from an almost uniform solution, the process first drifts towards the uniform unstable solution, $\rho_{\mathrm{e}}$. Close to $\rho_{\mathrm{e}}$, however, it diverges until one unit is empty and the other one bears all the load. The final density of this load-bearing unit, $\rho_{\mathrm{m}}$, can be derived from $\rho=U / k$, with $U$ corresponding to equation (13) (see Appendix). If the density of one element is zero, the other becomes

$$
\rho_{\mathrm{m}}=\left[\frac{P^{2}}{2 A^{2} c k}\right]^{1 /(\gamma+1)}=\rho_{\mathrm{e}}(4)^{1 /(\gamma+1)}
$$

Hence, the total mass of the nonuniform solution $\left(\rho_{\mathrm{m}}\right.$ for one unit and the other unit completely resorbed) is a factor $\frac{1}{2}(4)^{1 /(\gamma+1)}$ of the uniform unstable solution ( $\rho_{\mathrm{e}}$ for both units). With $\gamma=2$, this factor is 0.79 , indicating that the nonuniform solution contains only $79 \%$ of the total bone mass of the uniform solution.

Three alternatives for the remodeling stimulus $S$ were tested for their stability behavior: (a) based on SED, $S=U^{n} \rho^{m}$; (b) based on the stress, $S=\sigma^{n} \rho^{m}$; (c) based on the strain, $S=\varepsilon^{n} \rho^{m}$. If these stimuli expressions are applied in equation (7), instead of $U / \rho$, the stability conditions for the uniform solution can again be calculated, following the method presented in the appendix.

Figure 8 shows the conditions for $m, n$ and $\gamma$ which provide a stable uniform ('continuous') solution (grey areas). In all cases $m<0$ is required. When this is the case, the stress stimulus always provides a stable uniform solution when $n<0$, whereas the strain stimulus does so for $n>0$, in both cases regardless of the value of $\gamma$. When $m<0$, the SED stimulus only provides
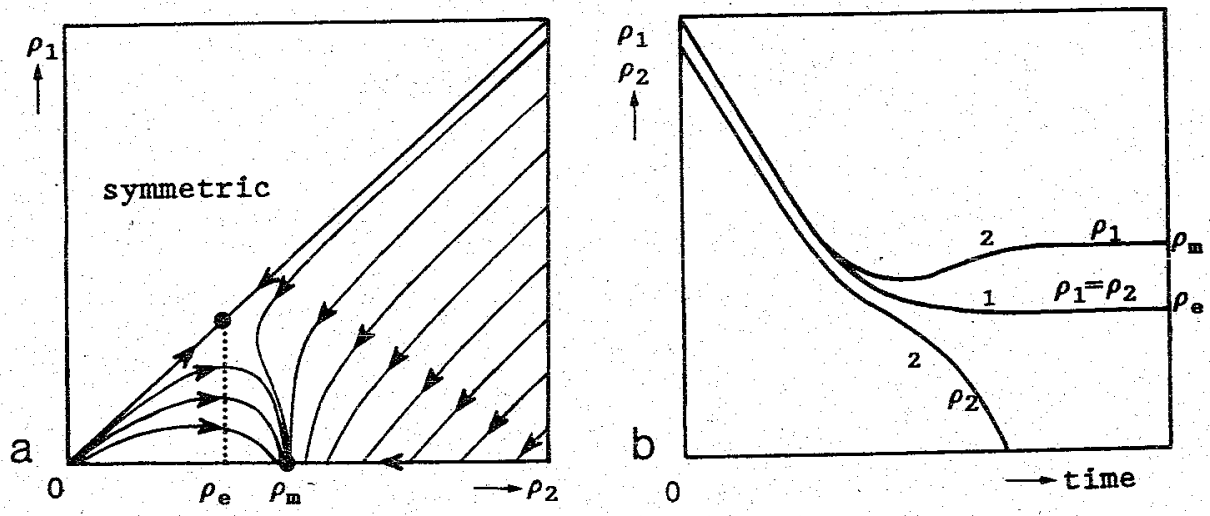

Fig 7. (a) Phase plot of the two-unit model with $\gamma=2.0$. The uniform solution, $\rho_{1}=\rho_{2}=\rho_{\mathrm{e}}$, is a saddle point. (b) The development of the density in two units during the process is shown. Line 1 indicates the convergence to the uniform solution (only in case the starting configuration is exactly at $\rho_{1}=\rho_{2}$ ) and lines 2 . indicate the process from a nonuniform starting configuration. 
stimulus stability
conditions (equation (13)) $\mathrm{m}<0$

$m^{2}-n^{2} \gamma^{2}>0$

$\mathrm{m}+1 / 2 \mathrm{n} \gamma<0$

$\mathrm{m}^{2}+\mathrm{mn} \boldsymbol{\gamma}>0$ stability area

in $n-m$ plane
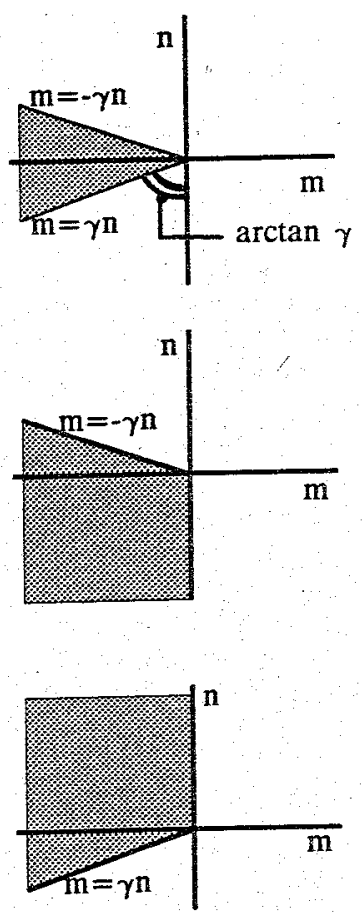

Fig. 8. The stability conditions for three different stimuli, all based on a combination of stress, strain and apparent density. The parameter $\gamma$ from the relationship $E=c \rho^{\gamma}$ is assumed to be always positive. The grey areas indicate stability for the uniform ('continuous') solution.

uniform stability while $\gamma<m /|n|$. A realistic value for $\gamma$ is in the range 2-3 (Rice et al., 1988; Hodgskinson and Currey, 1989). For example, if $\gamma=3$ and $n=1$, $m<-3$ is required for stability in the case of the SED and the stress stimuli (e.g. $S=U / \rho^{4}$ or $S=\sigma / \rho^{4}$ ). If $\gamma=2$ and $n=1$ the stimuli $S=U / \rho^{3}$ and $S=\sigma / \rho^{3}$ would provide stability in both cases (the strain stimulus always provides uniform stability while $n>0$ ). If " $n$ is increased to $>1, m$ must also be increased in absolute value if stability is to be obtained for $y>2$. It should be observed that the present two-unit model is probably not a representative one to investigate the strain stimulus, because the basic assumption of this model is that $\varepsilon_{1}=\varepsilon_{2}=\varepsilon$.

\section{The plate model}

After five increments the simulation analysis with $5 \times 5$ elements (Fig. 3 ), started from a uniform density distribution, produces a configuration with a density distribution such that the objective function is almost satisfied (Fig. 9). The density distribution is precisely as expected [Fig. 9(a)]: an increasing density from right to left. All stimuli values in the sensor points are between 0.19 and $0.28 \mathrm{~J} \mathrm{~g}^{-1}$, which is relatively close to the reference value $k=0.25 \mathrm{~J} \mathrm{~g}^{-1}$ [Fig. 9(b)]. Nevertheless, the total objective function $\Theta$ is not exactly zero and convergence is not obtained as yet.
The iterative procedure does not converge to the continuous end configuration, but it diverges from the continuous solution, which it had approached already so closely, until another density distribution is found which is not smooth, but discontinuous [Fig. 10(a)]. At locations where the density did not reach the upper boundary $\left(1.73 \mathrm{~g} \mathrm{~cm}^{-3}\right)$ or the lower boundary $\left(0.01 \mathrm{~g} \mathrm{~cm}^{-3}\right)$, the stimulus is exactly $0.25 \mathrm{~J} \mathrm{~g}^{-1}$, indicating that the objective, $U / p=k$, is satisfied [Fig. 10(b)]. One element at the upper corner' on the right did not converge [Fig. 10(b)] and continued to alternate its stimulus value, which is believed to be an insignificant phenomenon, related to the end effect of the mesh in combination with the small load at that location. The convergence of this element did improve, however, when the time step was further reduced.

With the use of higher-order elements (eight nodes and a quadratic displacement field), or when the stimulus value in the $5 \times 5$ mesh was sampled as the average from four or 16 subelements (Fig. 3), a similar result is found, consistently converging in a stable, discontinuous end configuration. Reducing the size of the time step further does not have any influence on this result, except for the alternating element in the upper right-hand corner. This clearly demonstrates again that the discontinuity does not occur because of 


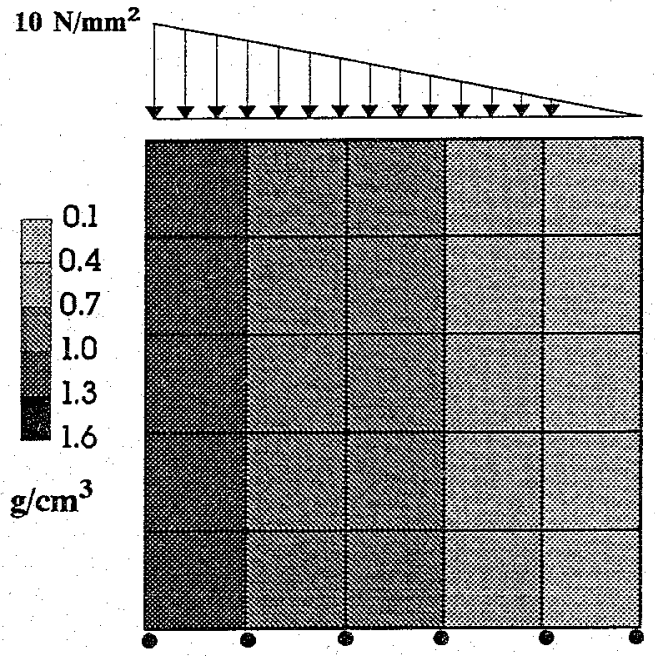

a) APPARENT DENSITY

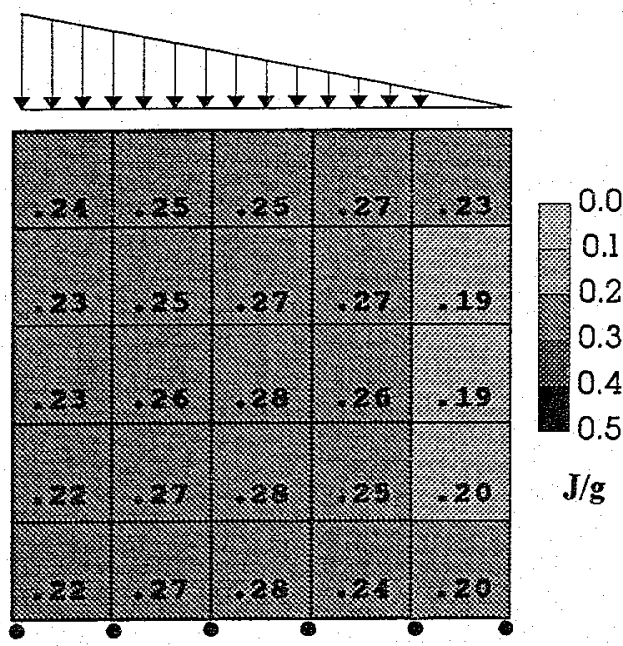

b) STIMULUS

Fig. 9. The apparent density distribution $\rho$ (a) and the stimulus (strain energy per unit of bone mass), $U / \rho$ (b) after five iterations of the remodeling process of the plate with $5 \times 5$ elements, with $\gamma=2.0$. The initial density distribution was $0.8 \mathrm{~g} \mathrm{~cm}^{-3}$ for all elements. The reference stimulus value, $k=0.25 \mathrm{Jg}^{-1}$, is closely approximated by all elements, as indicated in (b).

$10 \mathrm{~N} / \mathrm{mm}^{2}$

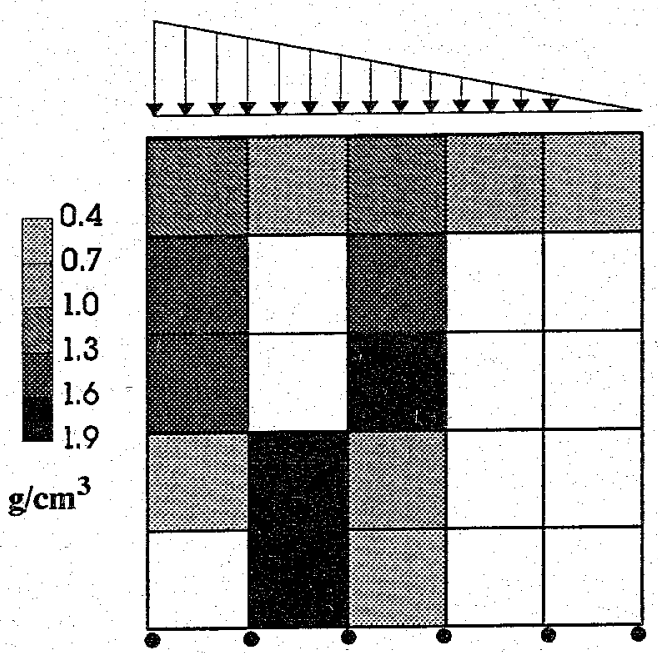

a) APPARENT DENSITY
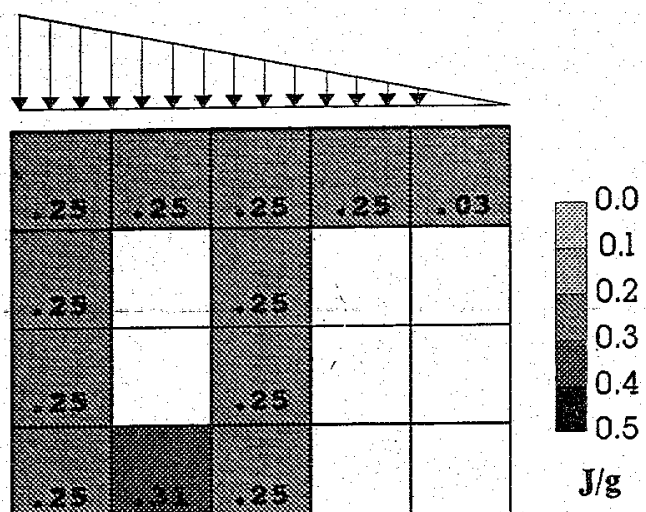

$\mathrm{J} / \mathrm{g}$

Fig. 10. The apparent density distribution, $\rho(\mathrm{a})$ and stimulus (strain energy per unit of bone mass), $U / \rho$ (b) in the end configuration of the $5 \times 5$ elements plate, with $\gamma=2.0$. Except for the one element in the upper right, all elements have reached the objective $U / \rho=0.25 \mathrm{Jg}^{-1}$ or have reached the boundary density value $\left(\rho_{\mathrm{sb}}=1.74 \mathrm{~g} \mathrm{~cm}^{-3}\right.$, or complete resorption, $\left.\rho=0.01 \mathrm{~g} \mathrm{~cm}^{-3}\right)$.

an imprecise stress/strain calculation in the FEM procedure, but originates in the description of the remodeling process, especially by the behavior of the relation $E=c \rho^{\gamma}$ in this process.

In the two-unit model it was found that for $\gamma<1.0$ the uniform solution was stable. For the square-plate model the process also converges to the continuous solution when $\gamma<1.0$. Figure 11 shows a smooth continuous end configuration with this model for $\gamma=0.5$.

The results of three mesh refinements, again with $\gamma=2$, are shown in Fig. 12. The end configurations obtained are quite remarkable. A highly ordered 


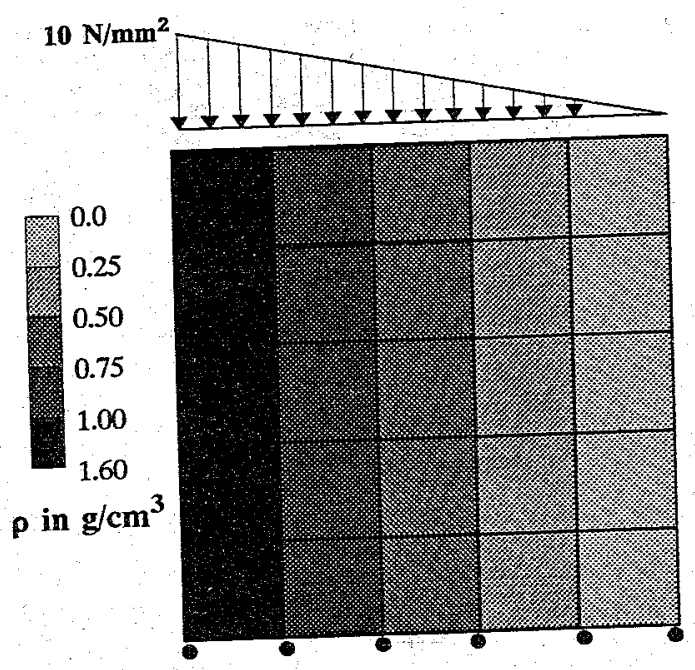

Fig. 11. The apparent density of the $5 \times 5$ elements mesh after remodeling with the relationship: $E=100 \rho^{0.5}(\gamma=0.5)$. Because the power index $\gamma$ is less than 1.0 the continuous solution has become stable. The stimulus, $U / \rho$, has reached exactly the reference stimulus value of $0.25 \mathrm{Jg}^{-1}$ in all elements.

structure is found in the direction of the support, ending in columns of a certain thickness. The general trend of the $5 \times 5$ mesh is confirmed in the more refined meshes. It seems that a more refined mesh is capable of showing more details of a repetitive composition. Intermediate results during the iterative process did show that all simulations passed the smooth, continuous solution closely, just like the $5 \times 5$ mesh in Fig. 9. The results of these four simulations after interpolation with the postprocessor are shown in Fig. 13. For the coarse meshes [Fig. 13(a) and (b)] the interpolated result does not contain any voids. These were smoothed out by the post-processor. The interpolated result of the most refined mesh [Fig. 13(d)] looks very similar to the result without interpolation [Fig. 12(d)]. Again, the general trends of the $40 \times 40$ elements mesh [Fig. 13(d)] can be found already in the coarse $5 \times 5$ elements mesh of Fig. 13(a).

Figure 14 shows the development of the total mass of the structure during the simulatioin process with the finest mesh [Fig. 12(d)]. This process had passed the smooth continuous solution closely in increment eight. The mass in the end configuration (from increment 40 and onwards) is approximately $20 \%$ lower than in increment eight. This indicates that the unstable continuous solution contains more mass than the discontinuous end configuration, similar to the mass reduction found in the two-unit model. The stimulus values in this end configuration, however, are not really close to the reference stimulus $k$. This is compatible with the result of Fig. 10(b), where it was shown that once the density of an element has reached the maximum value $\rho_{\mathrm{cb}}$, the stimulus usually overshoots the reference value. In the end configuration of the finest mesh [Fig. 12(d)], for example, this over- shoot is shown in Fig. 15. The stimulus values for those elements which are not resorbed are all in the range $0.25-0.5 \mathrm{Jg}^{-1}$, which is not extremely high relative to the reference value of $0.25 \mathrm{Jg}^{-1}$.

\section{DISCUSSION}

If we take the prediction of femoral bone morphology in terms of density patterns, optimized according to the remodeling rule of equation (3), at face value (Fig. 5), its similarity to reality is surprisingly good, despite the fact that the FE model used is based on a number of assumptions and simplifications. The model is two-dimensional, the number of (static) loading cases is limited to only three, and the modulus-density relationship $E=c \rho^{3}$ used is a rather crude approximation, with the cubic exponent probably somewhat too high (Rice et al., 1988). In addition, the choice of strain energy as the remodeling stimulus was more or less based on the fact that it is an easily interpretable physical scalar which is related to both stress and strain. The existence of strain energy sensors have never been documented in experiments. However, the similarity to reality obtained in the prediction seems even better than that found by Carter et al. (1989) and by Beaupré et al. (1990b): We have little doubt that this is due to the inclusion of the side plate in our FE model, as opposed to the model of these authors, and to the formulation of the remodeling rule as a time process, as opposed to Carter et al. (1989), since these are the only differences between our model and theirs. Hence, although both the stimulus and the remodeling rule selected are purely hypothetical, and the possibility that other stimuli or remodeling rules would work equally well cannot be discarded, the results obtained are very satisfactory. However, the solution process, in fact, produces a discontinuous patchwork of element density values, whereby the similarity with the density distribution of the real femur is found only in the mass distribution over a certain region, containing at least a few elements, which appears after interpolation by the postprocessor.

The analysis of the two-unit model provided an explanation for this behavior. It turned out that, when in the relation $E=c \rho^{\gamma}, \gamma>1$, the continuous solution is an unstable 'saddle point' while the stable solution is one in which each element tends to converge to either cortical bone or nothing. This explanation, suggested by the two-unit model, was indeed confirmed by the simplified plate model: taking $\gamma<1 \quad(\gamma=0.5$, Fig. 11) yields a continuous solution; taking $\gamma>1[\gamma=2$, Fig. $12(a-d)]$ leads to a discontinuous patchwork. Evidently, a value of $\gamma<1$ seems unrealistic according to experimental data reported in the literature (Rice et al., 1988; Hodgskinson and Currey, 1988), suggesting a value between 2 and 3 . In fact, Williams and Lewis (1982) reported a linear relationship between area 


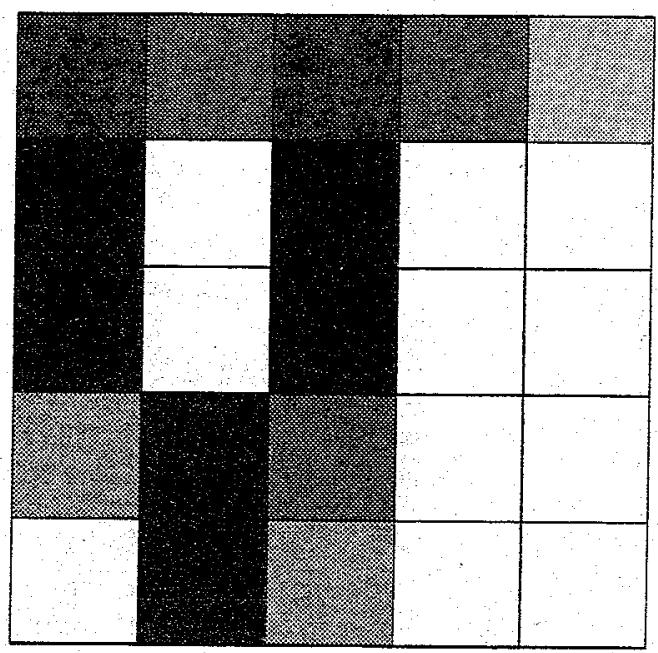

(a)

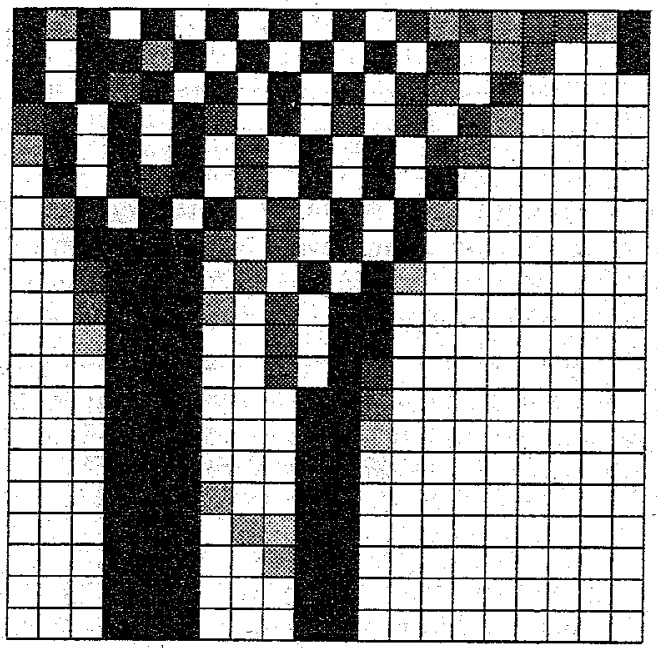

(c)

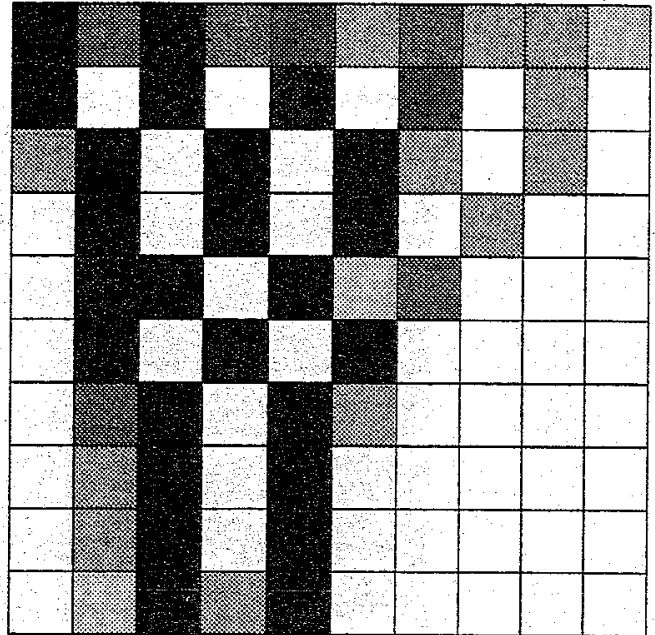

(b)

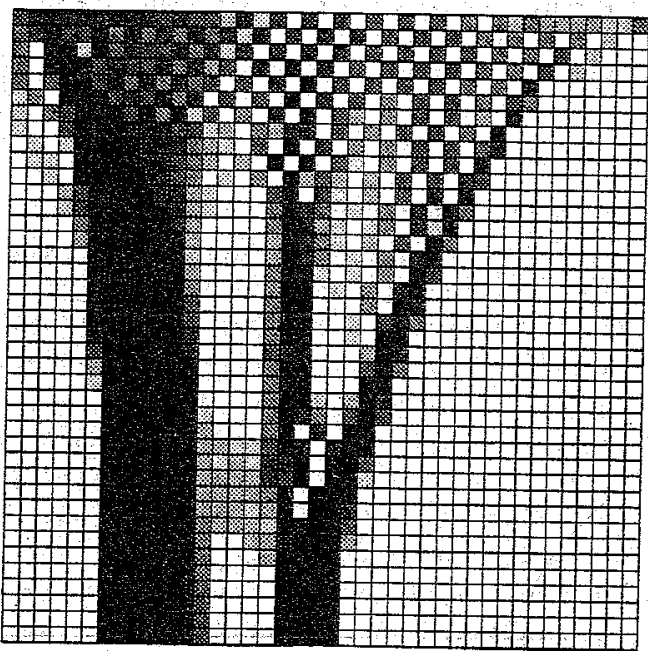

(d)

Fig. 12. Density distribution $\left(\rho\right.$ in $\left.\mathrm{g} \mathrm{cm}^{-3}\right)$ after remodeling of the plate problem with $5 \times 5,10 \times 10,20 \times 20$ and $40 \times 40$ elements (sensors), with $\gamma=2.0$.

fraction and elastic modulus (hence, $\gamma \approx 1.0$ ), based on experiments with cancellous bone from the upper region of a tibia. However, their density range was quite narrow; hence, it would not be surprising if there is little statistical difference between a linear and a power fit of their data. Whether the continuous solution in our two-unit model is stable for $\gamma=1.0$ is not certain. We did find, however, that when $\gamma$ approaches 1.0 from below, the time to reach the continuous solution becomes infinite.

At this point one may ask the question whether we really want a continuous solution. This brings us to a paradox: we use a model in which a discontinuous structure (trabecular bone) is represented as a continuous material, the remodeling simulatioin is applied and the result is not, as we expect, again a con- tinuous material as in the original model, but rather a discontinuous one as in reality! Intuitively, it seems that one could not consider a material as continuous and discontinuous in one and the same analysis (Harrigan et al., 1988).

The nirst obvious question is whether this behavior is due to the FE-formulation of the model, or to the model of the remodeling process itself. In order to investigate this question, several tests were performed. Higher-order elements with more nodes were used and the mesh density was varied. The iterative feedback procedure was executed per integration point, instead of per element, and also per group of elements. None of these variations would change the characteristics of the solution, which led us to investigate the simulation procedure more fundamentally in the plate 


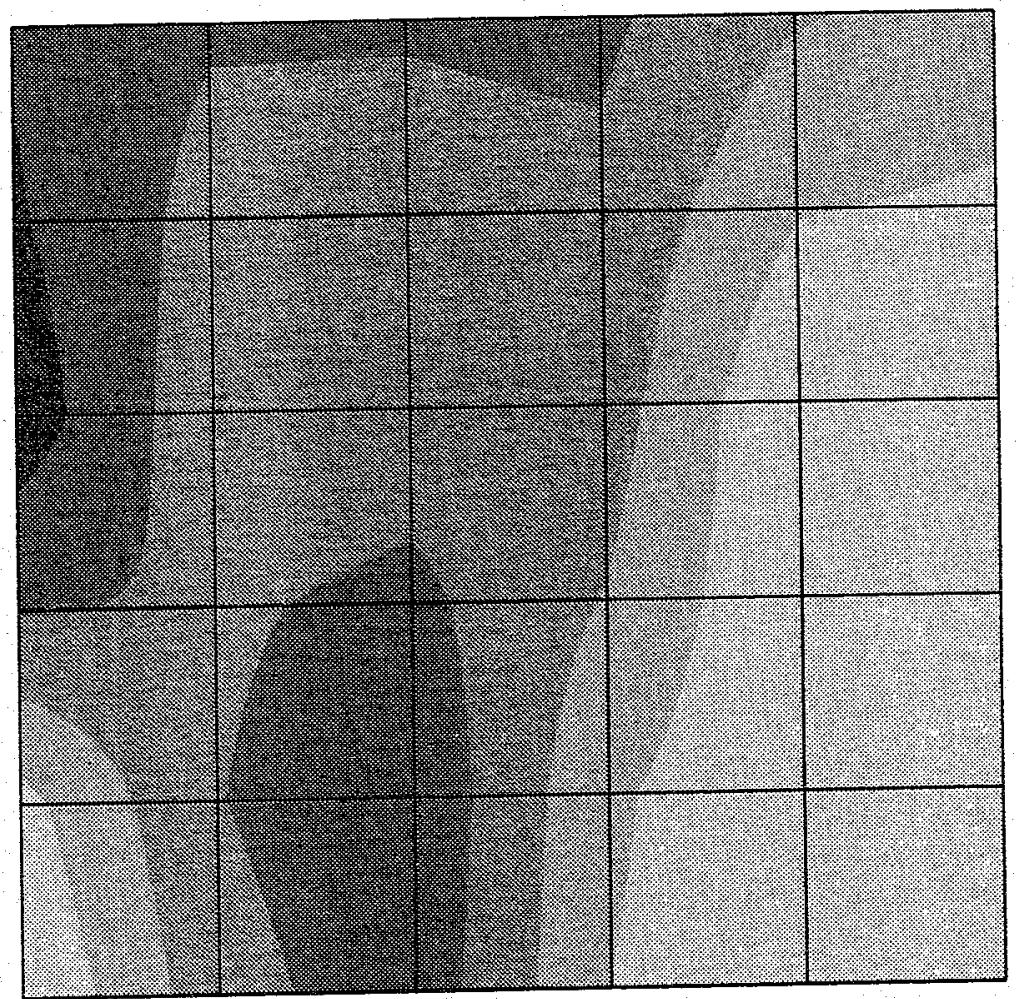

(a)

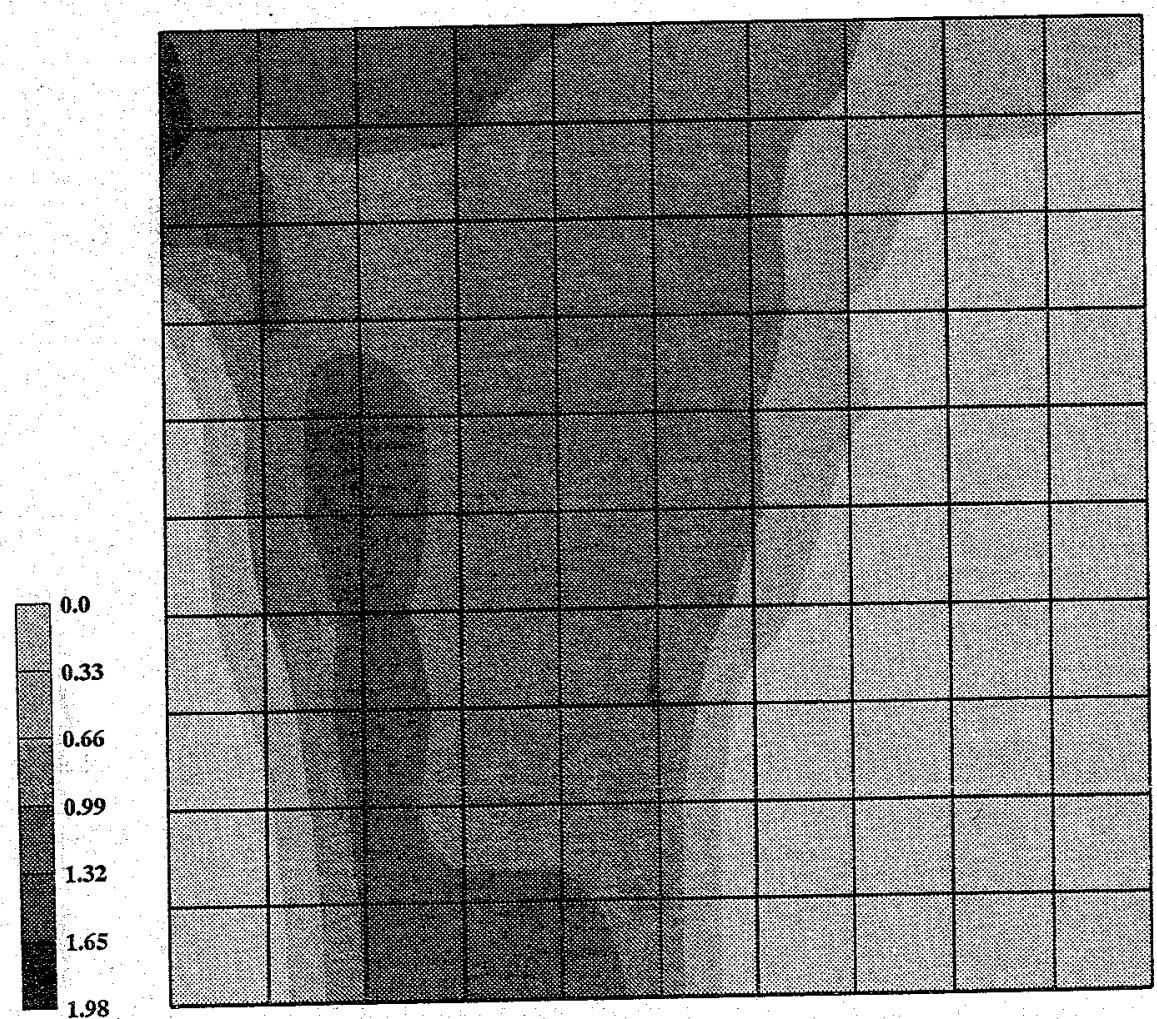

$\rho$ in $g / \mathrm{cm}^{3}$

(b)

Fig. 13(a,b). 


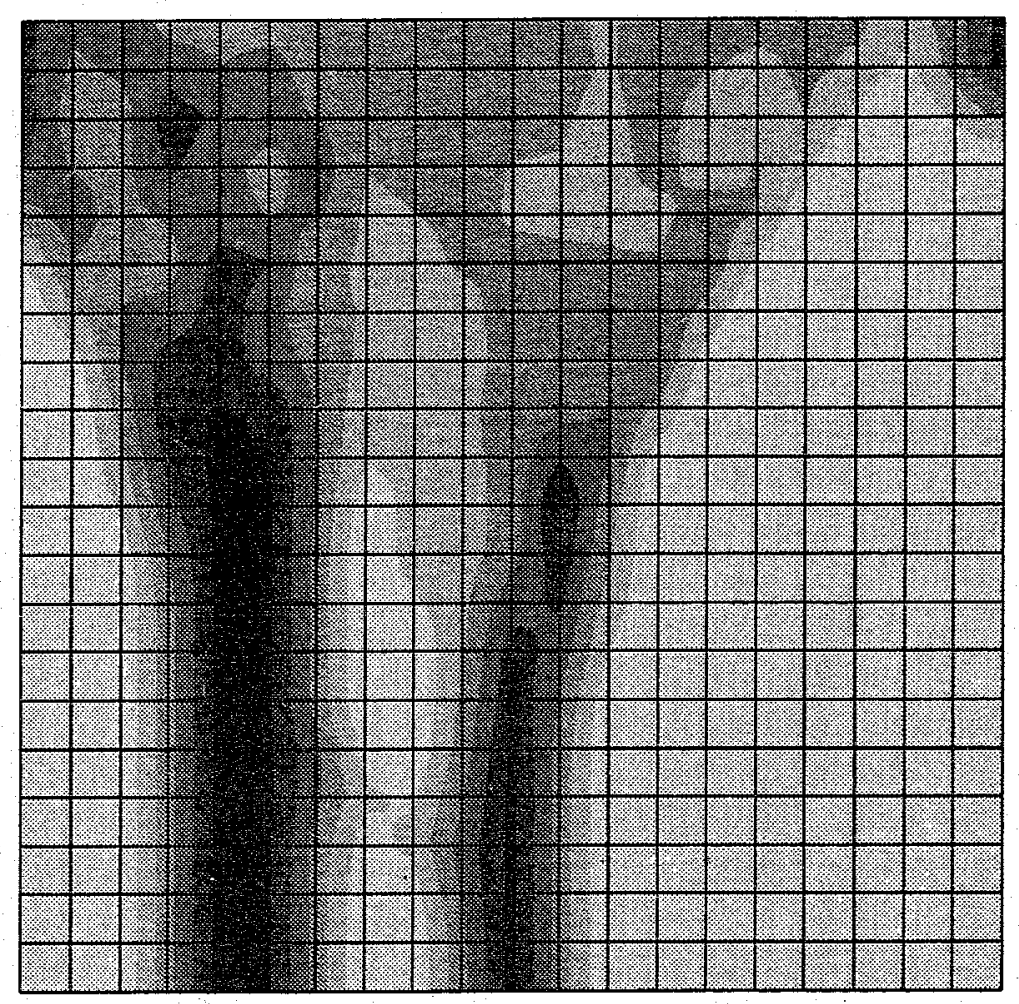

(c)

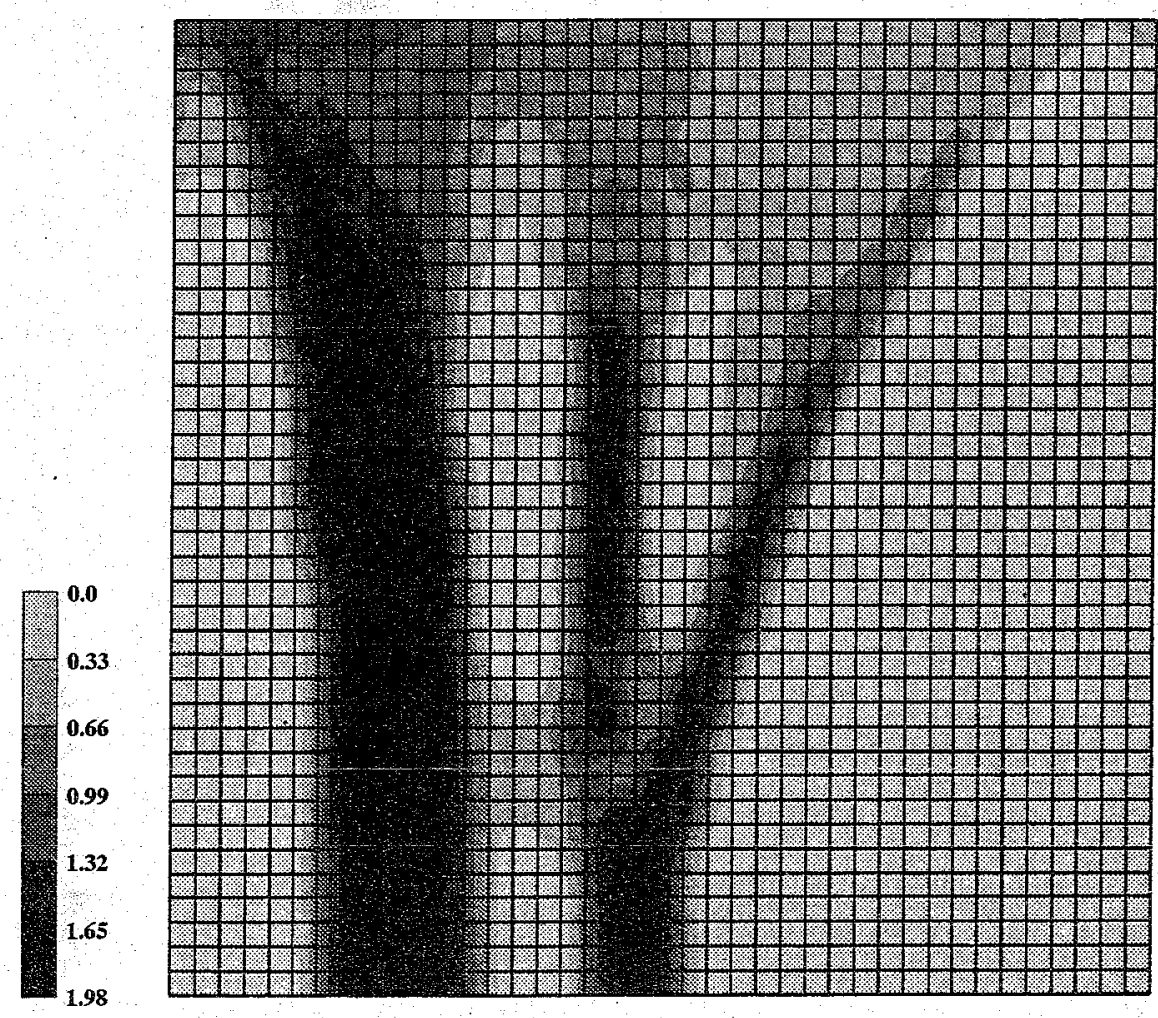

$\rho$ in $\mathrm{g} / \mathrm{cm}^{3}$

(d)

Fig. 13. Density distribution ( $\rho$ in $\mathrm{g} \mathrm{cm}^{-3}$ ) after remodeling of the plate problem $(\gamma=2.0)$ with different element sizes, after interpolation with the postprocessor. 


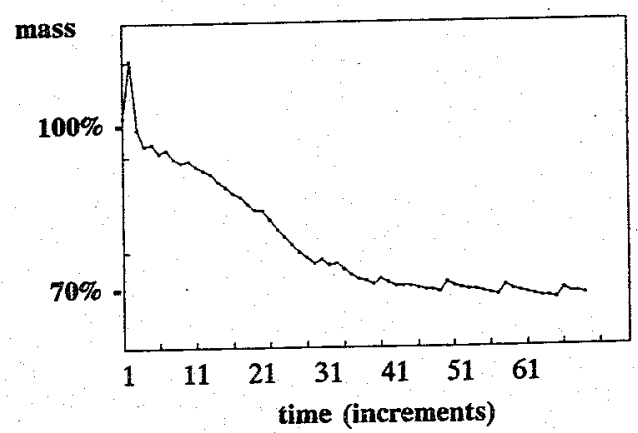

Fig. 14. Mass change during the remodeling process of the plate problem with the finest mesh [Fig. 12(d)]. The initial mass is indicated as $100 \%$. The continuous solution is approximated closely after six to ten increments. The total mass reduction relative to the initial situation is about $25 \%$ and the mass difference between the approximated continuous solution (eighth iteration) and discontinuous end configuration is about $20 \%$.

and two-unit models. We also found out that the effects obtained were not unique. Cowin and Nachlinger (1978) showed with a stability analysis that the adaptive elastic boundary-initial-value problem is, in principle, strongly dependent on the remodeling rate equation and one can easily construct a process with discontinuous solutions. In global FE-optimization studies of technical structures in the field of composite-material design (Strang and Kohn 1986; Bendsoe and Kikuchi, 1988) also discontinuous results were reported. Goldstein et al. (1990) used a similar optimization scheme to predict bone-ingrowth patterns around porous-coated implants, with similar results. It appeared that the optimization procedure preferred a geometrical solution (a discontinuous patch work) to a material one (a continuous solution of nonuniform elastic modulus distributions).

Of course, it is possible to use additional constraints for the optimization procedure, to force the solution towards a less discontinuous one. Although this is fine for optimization of engineering structures (Strang and Kohn, 1986), it is difficult to see what such a constraint would represent in terms of the biological process. Such a measure would, in our case, adapt the model to the FE-formulation instead of the opposite. It is quite trivial to see that if one would apply global optimization to the two-unit model, for example, by minimizing

$$
\sum_{i=1}^{2}\left[\frac{U_{i}}{\rho_{i}}-k\right]
$$

one obtains precisely the (stable) continuous solution $\left(\rho_{1}=\rho_{2}\right)$. If a similar (global) optimization scheme is applied to the proximal femur, the result is not a discontinuous patchwork but rather a nonuniform distribution of densities (Kuiper et al., 1991). However, such a global optimization scheme is not a model for a biological self-optimization process.

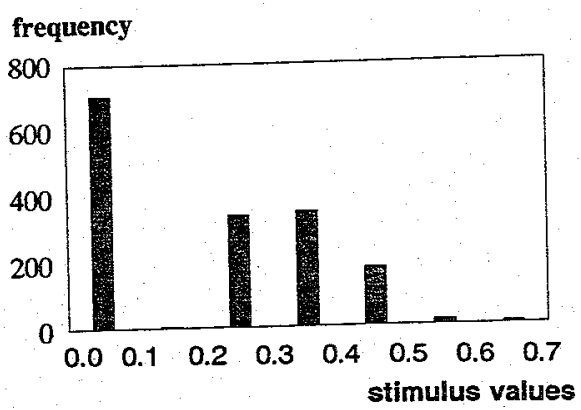

Fig. 15. The distribution of the stimulus values $(U / \rho)$ after remodeling of the plate problem with 1600 elements [Fig. 12(d)]. About 700 elements have become empty and approximately 900 elements are filled with bone, which have a stimulus value between one and two times the reference a stimulus value between one and two times
stimulus value $k=0.25 \cdot \mathrm{Jg}^{-1}$.

The second question is whether we could repair the model in such a way that we can work with apparent quantities throughout the whole analysis without violating the concept of bone as a self-optimizing material while producing results of the same realistic quality. Candidates for repair are the stimulus characteristics, the relationship between elastic modulus and apparent density and the relationship between tissuelevel quantities and apparent quantities in general. The analysis of the two-unit model indicated that an alternative choice for the stimulus would not improve the model in this respect. However, this simple model is not really suitable to investigate a strain-based stimulus, due to the constraint condition of equal strain in the two units. It would also be possible to assume a nonlinear instead of a linear relationship between stimulus and response, including, for instance, a 'dead zone' or a 'lazy zone' (Weinans et al., 1991). Our experience with such a relationship is that it does attenuate the patchwork effect but does not prevent it. The matter of the modulus/density relationship, the value of the exponent $\gamma$, was already discussed above. Where the continuum assumptions, in general, are concerned, there are several options to consider. First of all, one could represent trabecular bone as a discontinuous structure to begin with, using micro-FE models of the structure itself. In that case, the concept of 'internal remodeling' could be discarded, in favor of 'external' or 'surface modeling' only, which would, indeed, bring us much closer to the biological process as it occurs in reality, but would also remove us far from the context of the present model. In other words, this would be a totally different ball game and, presently, rather impractical in view of the computer capacity it requires. Secondly, the directionality of the trabecular structure could be accounted for in apparent anisotropy of the continuum model, in conjunction with an appropriate remodeling formulation. Fyhrie and Carter (1986) attempted to incorporate such a formulation, but this was not worked out further. Such a solution may be 
feasible, but its formulation is not trivial, and it is doubtful, we believe, that it would solve the present problem, for reasons explained below. Then finally, there may be a better way to relate tissue-level quantities to apparent ones, for instance, by applying homogenization theory (Hollister et al., 1989). Again, at this moment it is uncertain whether that would produce results of a different nature.

The possibility that a satisfactory model formulation which allows for a continuous representation of trabecular bone and the remodeling process can be found cannot be discarded at this point in time. We believe, however, that the results described here are inherent to the concept of bone as a self-optimizing material, rather than to the way in which this concept is described in a model. This concept implies that bone mass is regulated by multiple local units, which work independently. If the control mechanism in the simulation model is characterized by a self-enhancing system (a positive feedback), then the occurrence of discontinuities is inevitable. Since this positive feedback loop is central to the concept of self-optimization itself, where more mass will attract more load and vice versa, it is difficult to see how one could get away from it in a model, without sacrificing this concept.

When we consider the simulation process from another angle, we find that it is governed by a set of nonlinear differential equations, the number of which is equal to the number of elements (or sensor points). Such a process is liable to chaotic behavior and the solution may have an irregular structure, which may be described by a fractal (Yates, 1987; Wlczek, 1991). The two-unit model already clarifies that the solution is very susceptible to small deviations in the initial conditions: which one of the two elements turns empty depends on only virtual differences in initial stimulus values. When we have a complex multiple element (or sensor) system, the effect of this sensitivity multiplies and the solutions become truly chaotic. Strangely enough, however, when we let the proximal femur model of Fig. 2 remodel from totally different initial density distributions, obtained from stochastic Monte Carlo generation (Weinans et al., 1989), the final solutions are very similar, but not equal. Hence, the process seems to be one of ordered chaos, displaying self-organizational qualities towards an attractor. As we have seen in the plate problem, the stimulus values in those elements which still contain bone mass in the end configurations are never far removed from the objective $k$, although there is no direct constraint for that in the formulation. Hence, the term 'attractor state' for the end configuration of the remodeling simulation process seems to be right on the mark (Carter et al., 1989): We have also found (Weinans et al., 1989) that the self-organization process towards similar end configurations only occurs when the values of the physical parameters chosen are within particular boundaries. When we consider the two-unit and the plate models, we also find that the stable, discontinuous solutions contain more mass than the unstable, continuous one. Hence, minimal mass seems to be another (global) objective or attractor of the process.

The relationship with chaotic phenomena (Yates, 1987; Kauffman, 1986) and fractals is also suggested in the solutions of the plate model for varying mesh densities (Fig. 12). Here we find the geometric structure and self-similarity typical of a fractal (Mandelbrot, 1983; Bittner and Sernetz, 1990; Wlczek, 1991). We see that the geometric configuration converges when mesh density increases, but that the first coarse mesh [Fig. 12(a)] already holds the general features of the refined one [Fig. 12(d)]. It is also seen that the similarity between the patchwork (Fig. 12) and the interpolated results (Fig. 13) increases when mesh density increases. It is not difficult to predict what would happen if the mesh were further refined, using a thought experiment: in Fig. 12(c), for example, one can find a region of $5 \times 5$ elements, the structure of which is very similar to the whole of Fig. 12(a). If we were to refine the mesh to a total of $320 \times 320$ elements, that particular area would have $40 \times 40$ elements, as in the total mesh of Fig. 12(d). In the same way that the configuration of Fig. 12(a) develops into the one of Figure 12(d), our particular area would develop in a structure similar to the whole of Fig. 12(d). Hence, when mesh density is increased, more self-similarity will be seen, a larger part of the structure will have developed into the discontinuous patchwork, by which is meant that the solution becomes independent of the size of the element. The validity of the FE-representation of such a structure depends on the mesh refinement per strut. Since bilinear elements are used, at least two elements over the thickness of a strut are required to obtain a reasonable interpretation of the stress field in the strut. It is also demonstrated that a relatively coarse mesh cannot predict the morphology in detail; however, it can represent the mass distribution over large areas reasonably well. Hence, there is no reason to dismiss the theory as applied to the proximal femur as invalid or useless.

Our little thought experiment signifies that mesh refinement produces a fractal-like self-similar trabecular structure, of which the FE-description would be a valid one, apart from a small region close to the boundary. This small region would approach a zero thickness when the number of elements approaches infinity. It is intuitively obvious that for an adequately refined mesh, in which the dependence of element dimensions is restricted to small parts only, the geometric characteristics of the solution would depend only on the characteristics of the loads, the value of the objective $k$ and the maximal attainable trabecular elastic modulus (hence, the maximal trabecular density, or rather the degree of mineralization). This brings us to a whole new interpretation of the plate analysis. Let us assume that we apply the continuum 
assumption on a lower level, that of the trabecular tissue. Replacing the apparent density $\rho$ by the tissue density $\rho_{t}$ (degree of mineralization), the apparent SED $U$ by the tissue SED $U_{t}$ (and $S$ by $S_{t}$ ), and requiring that, starting from a uniform density domain, each sensor point (or element) organizes its own mineralization $\rho_{\mathrm{t}}$ between zero and a maximum, we have an analogous problem description as in the plate model. Since Currey (1988) showed that $E_{\mathrm{t}}=c^{\prime} \rho_{\mathrm{t}}^{\gamma}$, where $\gamma \approx 3$ (hence, $\gamma>1$ ), we will also find a similar (patchy) solution. Those who might argue that $S_{\mathrm{t}}=U_{\mathrm{t}} / \rho_{\mathrm{t}}=$ constant would not be a sensible criterion in this case are free to substitute $S_{\mathrm{t}}=U_{\mathrm{t}}=$ constant as the optimization goal: as shown here the result would not be principally different; a trabecular-like structure would result. As long as the mesh would be fine enough, i.e. more than two elements over the width of the struts that would emerge (for instance, more elements per sensor point), the FEM would be a reasonable accurate description of this discontinuous trabecular structure. Evidently, the precise morphology and dimensional characteristics of the emerging trabecular-like structure depends only on the characteristics of the load, the maximal tissue mineralization (or rather the maximal value of $\rho_{t}$ ), the value of $k$ and on the sensor density. This is a thought worth contemplating. It means that the assumption of bone as a self-optimizing material, made operational in a computer-simulation model, produces precisely what we have: a discontinuous, trabecular structure. This idea is, in fact, not so different from some of the original thoughts of Roux (1881) and Wolff (1892), as indicated by the following citation of Wolff: . . . alterations of the internal structure clearly observed and following mathematical rules, as well as secondary alterations of the external form of bones following the same mathematical rules, .... The basic concept of self-optimization was already indicated by Roux, who called it 'direct functional self-shaping' (as cited by Wolf, 1892). The principle of this concept was explained by Roux as a struggle of the elements: 'The cells compete with each other, the tissues made of these cells compete, the organs made of these tissues compete and the groups of organs compete', and 'A functional structure develops as a consequence of the victory of the most qualified elements, ... . Roux believed that these victory elements become a bony trabeculae which unloads its immediate surrounding.

Based on these results and reflections, we hypothesize (i) that bone is indeed a self-optimizing material which produces a self-similar trabecular morphology, a fractal, in a chaotic process of self-organization, whereby uniform SED per unit mass, or a similar mechanical signal, is an attractor, (ii) that the morphology has qualities of minimal weight, and (iii) that its morphological and dimensional characteristics depend on the local loading characteristics, the maximal degree of mineralization, the sensor density and on the attractor value. This implies that all characteristic morphological differences between location and species could be explained by a variation in the abovementioned parameters.

Acknowledgement-Sponsored in part by the Netherlands Organization for Research (NWO/Medigon).

\section{REFERENCES}

Bendsoe, M. P. and Kikuchi, N. (1988) Generating optimal topologies in structural design using a homogenization method. Comp. Meth. Appl. Meth. Engng 71, 197-224.

Beaupré, G. S., Orr, T. E. and Carter, D. R. (1990a) An approach for time-dependent bone modeling and remodeling - theoretical development. $J$. orthop. Res. 8, 651-661.

Beaupre, G. S., Orr, T. E. and Carter, D. R. (1990b) An approach for time-dependent bone modeling and remodeling -application: a preliminary remodeling simulation. J. orthop. Res. 8, 662-670.

Bittner, H. R. and Sernetz, M. (1990) Selfsimilarity within limits: description with the log-logistic function. In Proc. 1 st IFIP Conference on Fractals. June 6-8, 1990, Lisbon, (Edited by Peitgen, H. O., Henriques, J. M. and Penedo, L. F.). Elsevier, Amsterdam.

Carter, D. R. and Hayes, W. C. (1977) the behavior of bone as a two-phase porous structure. J. Bone Jt Surg. 59-A, 954-962.

Carter, D. R., Orr, T. E. and Fyhrie, D. P. (1989) Relationships between loading history and femoral cancellous bone architecture. J. Biomechanics 22, 231-244.

Cowin, S. C. (1986) Wolff's law of trabecular architecture at remodeling equilibrium. J. biomech. Engng 108, 83-88.

Cowin, S. C. and Hegedus, D. H. (1976) Bone remodeling I: a theory of adaptive elasticity. J. Elasticity 6, 313-326.

Cowin. S. C. and Nachlinger, R. R. (1978) Bone remodeling III: uniqueness and stability in adaptive elasticity theory. J. Elasticity 8, 285-295.

Currey, J. D. (1988) The effect of porosity and mineral content on the Young's modulus of elasticity of compact bone. J. Biomechanics 21, 131-139.

Frost, H. M. (1964a) The Laws of Bone Structure. Charles C. Thomas, Springfieid, IL.

Frost, H. M. (1964b) Mathematical Elements of Lamellar Bone Remodeling. Charles $\mathrm{C}$. Thomas, Springfield, IL.

Frost, H. M. (1987) Vital biomechanics. Proposed general concepts for skeletal adaptations to mechanical usage. Calcif. Tissue Int. 42, 145-156.

Fyhrie, D. P. and Carter, D. R. (1986) A unifying principle relating stress to trabecular bone morphology. J. orthop. Res. 4, 304-317.

Fyhrie, D. P. and Carter, D. R. (1990) Femoral head apparent density distribution predicted from bone stresses. $J$. Biomechanics 23, 1-10.

Goldstein, S. A., Hollister, S. J., Kuhn, J. L. and Kikuchi, N. (1990) The mechanical and remodeling properties of trabecular bone. In Biomechanics of Diarthrodial Joints, Vol. II (Edited by Mow, V. C., Ratcliffe, A. and Woo, S. L.Y.), pp. 61-81. Springer, New York.

Harrigan, T. P., Jasty, M., Mann, R. W. and Harris, W. H. (1988) Limitations of the continuum assumption in cancellous bone. J. Biomechanics 21, 269-275.

Hart, R. T., Davy, D. T. and Heiple, K. G. (1984) Mathematical modeling and numerical solutions for functionaliy dependent bone remodeling. Calcif. Tissue Int. 36, S104-109.

Hegedus, D. H, and Cowin, S. C. (1976) Bone remodeling II small strain adaptive elasticity. J. Elasticity 6, 337-352. 
Hodgskinson, R. and Currey, J. D. (1990) Effects of structural variation on Young's modulus of non-human cancellous bone. Proc. Instn Mech. Engrs 204, 43-52.

Hollister, S. J., Fyhrie, D. P., Jepsen, K. J. and Goldstein, S. A. (1989) A new procedure for calculating apparent bone properties and tissue micro-stresses using homogenization theory. Trans. 35th orthop. Res. Soc. 14, 266.

Huiskes, R. (1980) Some fundamental aspects of humanjoint replacement. Acta orthop. scand. (Suppl.) 185.

Huiskes, R. and Chao, E. Y. S. (1983) A survey of finite element methods in orthopaedic biomechanics. J. Biomechanics 16, 385-409.

Huiskes, R., Weinans, H., Grootenboer, H. J., Dalstra, M. Fudala, B. and Sloof, T. J. (1987) Adaptive bone-remodeling theory applied to prosthetic-design analysis $J$. Biomechanics 20, 1135-1150.

Huiskes, R., Weinans, H., Summer, D. R., Fudala, B., Turner, H. J., Grootenboer, H. J. and Galante, J (1989a) Stressshielding, stress-bypassing and bone resorption around 'press-fit' and bone ingrowth THA. Trans 35th orthop. Res. Soc. 14, 529 .

Huiskes, R., Weinans, H. and Dalstra, M. (1989b) Adaptive bone remodeling and biomechanical design considerations for noncemented total hip arthroplasty. Orthopedics $12,1255-1267$.

Huiskes, R., Weinans, H., van Rietbergen, B., Sumner, D. R., Turner, T. M. and Galante, J. O. (1991) Validation of strain-adaptive bone remodeling analysis to predict bone morphology around noncemented THA. Trans 37 th orthop. Res. Soc. 16, 105.

Kauffman, A. (1986) Boolean systems, adaptive automata, evolution. In Disordered Systems and Biological Organization (Edited by Bienenstock, E., Fogelman Soulie, F. and Weisbuch, G.), NATO ASI Series F: Computer and Systems Sciences, Vol. 20. Springer, Berlin.

Kummer, B. K. F. (1972) Biomechanics of bone: mechanical properties, functional structure, functional adaptation. In Biomechanics: Its Foundation and Objectives (Edited by Fung, Y. C., Perrone, N. and Anliker, M.), pp. 237-271. Prentice Hail, Englewood Cliffs, NJ.

Kuiper, J. H., Huiskes, R. and Weinans, H. (1992) Bone remodeling: self-optimization vs. global optimization. Trans 38th orthop. Res. Soc. 17.

Mandelbrot, B. B. (1983) The Fractal Geometry of Nature. Freeman, New York.

Pauwels, F. (1965) Gesammelte Abhandlungen zur Funktionellen Anatomie des Bewegungsapparates. Springer, Berlin:

Rice, J. C., Cowin, S. C. and Bowman, J. A. (1988) On the dependence of the elasticity and strength of cancellous bone on apparent density. J. Biomechanics 21, 155-168.

Roux, W. (1881) As cited by Wolff (1892): Der Kampf der Theile im organismus. Engelmann, Leipzig.

Strang, G. (1986) Introduction to Applied Mathematics. Wellesley-Cambridge Press, Wellesley, MA.

Strang, G. and Kohn, R. V. (1986) Optimal design in elasticity and plasticity. Int. J. Num. Meth. Engng 22, 183-188.

Verdonschot, N. and Huiskes, R. (1990) FEM analyses of hip prostheses: validity of the 2-D side-plate model and the effects of torsion. In Proc. 7th Meeting of the Europe Society of Biomechanics, July 8-11, Aarhus, Denmark, p. A20.

Weinans, H., Huiskes, R. and Grootenboer, H. J. (1989) Convergence and uniqueness of adaptive bone remodeling. Trans. 35 th orthop. Res. Soc. $14,310$.

Weinans, H., Huiskes, R. and Grootenboer, H. J. (1990) A hypothesis concerning minimal bone density threshold levels as final stages of bone remodeling. Trans. 36 th orthop. Res. Soc. 15, 78.

Williams, J. L. and Lewis, J. L. (1982) Properties and an anisotropic model of cancellous bone from the proximal tibial epiphysis. J. biomech. Engng 104, 50-56.

Wlczek, P. (1991) Fractal analysis of blood vessels and bones. Workshop on fractal geometry and computer graphics, T. H. Darmstadt, University Bremen, 6-8 June 1991.

Wolff, J. (1892) Das Gesetz der Transformation der Knochen. Hirchwild, Berlin. (Translated as The Law of Bone Remodeling.) Springer, Berlin.

Yates, F. E. (1987) Control of self-organization. In Selforganizing Systems, the Emerge of Order (Edited by Yates, F. E.). Plenum, New York.

\section{APPENDIX}

The general feedback equation for the two-unit model is

$$
\begin{aligned}
& \frac{\mathrm{d} \rho_{1}}{\mathrm{~d} t}=B\left(S_{1}-k\right)=B\left(\frac{U_{1}}{\rho_{1}}-k\right)=F_{1}\left(\rho_{1}, \rho_{2}\right), \\
& \frac{\mathrm{d} \rho_{2}}{\mathrm{~d} t}=B\left(S_{2}-k\right)=B\left(\frac{U_{2}}{\rho_{2}}-k\right)=F_{2}\left(\rho_{1}, \rho_{2}\right) .
\end{aligned}
$$

With a set of initial conditions for $\rho_{\mathrm{i}}$ this differential equation can oe solved. From

$$
\varepsilon_{1}=\varepsilon_{2}=\varepsilon=\frac{P}{A\left(E_{1}+E_{2}\right)}
$$

with $A$ the area of cross section (Fig. 4),

$$
E_{1}=c \rho_{1}^{\gamma} \text { and } E_{2}=c \rho_{2}^{\gamma} \text {, }
$$

it follows that

$$
\varepsilon=\frac{P}{A c\left(\rho_{1}^{\gamma}+\rho_{2}^{\gamma}\right)}
$$

Additionally,

$$
U=\frac{1}{2} \sigma \varepsilon=\frac{1}{2} E \varepsilon^{2}
$$

so

$$
U_{1}=\frac{\rho_{1}^{\gamma} P^{2}}{2 A^{2} c\left(\rho_{1}^{\gamma}+\rho_{2}^{\gamma}\right)^{2}} \text { and } U_{2}=\frac{\rho_{2}^{\gamma} P^{2}}{2 A^{2} c\left(\rho_{1}^{\gamma}+\rho_{2}^{\gamma}\right)^{2}}
$$

If $\rho_{1}$ and $\rho_{2}$ are equal by definition (both sensors are coupled in that case) we can write for the equilibrium solution $\rho_{e}$

$$
\rho_{\mathrm{e}}=\rho_{1}=\rho_{2}=\frac{U_{1}}{k}=\frac{U_{2}}{k}=\frac{U_{\mathrm{e}}}{k} .
$$

With equation (13) this leads to

$$
\rho_{\mathrm{e}}=\left[\frac{P^{2}}{8 A^{2} c k}\right]^{1 /(\gamma+1)}
$$

This gives

$$
U_{\mathrm{e}}=\frac{P^{2}}{8 A^{2} c \rho_{\mathrm{e}}^{\gamma}}
$$

and the remodeling mechanism always leads to the equilibrium solution $\rho_{\mathrm{e}}$ as given in equation (14). Because a too high value for $\rho\left(\rho>\rho_{\mathrm{e}}\right)$ is always corrected adequately by a SED $U<U_{e}$; so $U / \rho$ in equation (9) is in that case always smaller than $k$, whereby $\mathrm{d} \rho / \mathrm{d} t$ becomes negative. In the same manner, a too small density always leads to a positive value of $\mathrm{d} \rho / \mathrm{d} t$, whereby again the appropriate correction is given. In case both sensors are not coupled and behave independently, it is difficult to determine the analytical solution. The 'optimal solution', however, is recognized as the uniform solution: $\rho_{1}=\rho_{2}=\rho_{\mathrm{e}}$. Hence, $\mathrm{d} \rho / \mathrm{d} t$ is exactly zero for both unit 1 and 2 . The stability of this solution can be analyzed by determination of the linearized set of differential equations around $\rho_{i}=\rho_{\mathrm{e}}$ [the linearized stability, Strang (1986)]. The coefficient matrix $\mathbf{C}$ of the linearized system $\left(\mathrm{d} \rho_{i} / \mathrm{d} t=C_{i j} \rho_{j}\right)$ 
is given by

$$
\mathbf{C}=\left[\begin{array}{ll}
\frac{\partial F_{1}}{\partial \rho_{1}} & \frac{\partial F_{1}}{\partial \rho_{2}} \\
\frac{\partial F_{2}}{\partial \rho_{1}} & \frac{\partial F_{2}}{\partial \rho_{2}}
\end{array}\right]
$$

Substituting equation (13) into equation (9) and differentiating with respect to $\rho_{1}$ or $\rho_{2}$ gives

$$
\frac{\partial F_{1}}{\partial \rho_{1}}=\frac{\partial F_{2}}{\partial \rho_{2}}=-\frac{B P^{2}}{8 A^{2} c} \rho^{-\gamma-2}
$$

$$
\frac{\partial F_{1}}{\partial \rho_{2}}=\frac{\partial F_{2}}{\partial \rho_{1}}=-\frac{\gamma B P^{2}}{8 A^{2} c} \rho^{-\gamma-2} .
$$

The matrix from equation (16) yields, therefore;

$$
\mathbf{C}=-\frac{B P^{2}}{8 A^{2} c} \rho^{-\gamma-2}\left[\begin{array}{ll}
1 & \gamma \\
\gamma & 1
\end{array}\right]
$$

The system described by the set of differential equation $\mathrm{d} \rho_{i} / \mathrm{d} t=C_{i j} \rho_{j}$ is only stable if both eigenvalues are negative. This means that trace $\mathbf{C}$ should be negative and the determinant of matrix $C$ should be positive, so $1-\gamma^{2}>0.0$. 